\title{
Optical ridge waveguides in Yb:YAG laser crystal produced by combi- nation of swift carbon ion irradiation and femtosecond laser ablation
}

\author{
Yazhou Cheng a , Jinman Lva ${ }^{a}$, Shavkat Akhmadaliev ${ }^{\mathrm{b}}$, Irene Hernández-Palmero ${ }^{\mathrm{c}}$, \\ Carolina Romero ${ }^{\mathrm{d}}$, Javier R. Vázquez de Aldana ${ }^{\mathrm{d}}$, Shengqiang Zhou ${ }^{\mathrm{b}}$, Feng Chen ${ }^{\mathrm{a}, *}$ \\ a Shandong University, School of Physics, State Key Laboratory of Crystal Materials, Jinan 250100, China \\ ${ }^{\mathrm{b}}$ Helmholtz-Zentrum Dresden-Rossendorf, Institute of Ion Beam Physics and Materials Research, Dresden, Germany \\ c Centro de Láseres Pulsados (CLPU), Parque Científico, Villamayor, Salamanca 37185, Spain \\ ${ }^{\mathrm{d}}$ Universidad de Salamanca, Laser Microprocessing Group, Salamanca 37008, Spain
}

\section{A R T I C L E I N F O}

\section{Article history:}

Received 16 December 2014

Received in revised form

13 March 2015

Accepted 31 March 2015

Available online 17 April 2015

Keywords:

Optical ridge waveguides

Swift ion irradiation

Femtosecond laser ablation

\begin{abstract}
A B S T R A C T
We report on the fabrication of optical ridge waveguides in ytterbium-doped yttrium aluminum garnet (Yb:YAG) single crystal by applying swift $\mathrm{C}^{5+}$ ion irradiation and the followed femtosecond laser ablation. The planar waveguide layer is first produced by $\mathrm{C}^{5+}$ ion irradiation and the laser ablation is used to microstructure the planar waveguide surface to construct ridge structures. The lowest propagation loss of the ridge waveguide has been determined to be $\sim 2.1 \mathrm{~dB} / \mathrm{cm}$. From the confocal micro-fluorescence and micro-Raman spectra obtained from the waveguide regions, the intensities, positions and widths of the emission-line peaks had no obvious changes with respect to those from the bulks, which indicate that $\mathrm{C}^{5+}$ ion irradiation does not affect the bulk-related properties of the Yb:YAG crystal significantly in the waveguide regions. The results obtained in this work suggest potential applications of the Yb:YAG ridge waveguides as integrated laser sources.
\end{abstract}

(c) 2015 Elsevier Ltd. All rights reserved.

\section{Introduction}

As the basic elements in integrated photonics and optical telecommunication systems, optical waveguides confine light fields within small volumes to dimension orders of a few microns, in which relatively high optical intensities could be reached compared to the bulk configurations [1]. In practice, the two-dimensional (2D) confined ridge waveguides are superior to the onedimensional (1D) planar partners owing to the more compact geometry and stronger spatial confinement of light fields [2]. Due to these properties, the $2 \mathrm{D}$ ridge waveguides can be used to construct more compact photonic devices [3], such as optical modulators [4], photo-detectors [5], and optical switchers [6]. Several techniques have been developed to fabricate optical waveguides in optical material, such as proton/ion exchange [7], metal diffusion [8], femtosecond (fs) laser ablation [9-12], and ion implantation/ irradiation [13-17].

The Yb:YAG crystal has been considered to be one of the most favorable laser-gain media for the solid-state lasers [18-23], mainly due to its intriguing advantages including outstanding fluorescence properties (long fluorescence lifetime, broad emission bands and large emission and absorption cross-section) [24,25], and lasing performance (high thermal conductivity and high mechanical stability) $[25,26]$.

As one of the most powerful techniques, the swift heavy ion irradiation (typically at energies higher than $1 \mathrm{MeV} / \mathrm{amu}$ ) has been extensively applied to fabricate waveguide owing to the reduced irradiation fluences and larger refractive index changes for waveguide formation [13]. The femtosecond laser ablation has become an increasingly attractive technique for microstructuring of a great variety of optical materials [27], owing to the various practical applications in ability for maskless material processing [28]. The combination of the ion irradiation and femtosecond laser ablation has been utilized to fabricate $2 \mathrm{D}$ ridge optical waveguide structures in laser and nonlinear crystals, in which enhanced features for both lasing and frequency doubling have been realized [29,30].

In this work, we have fabricated optical ridge waveguides in Yb:YAG crystals by combining swift carbon ion irradiation and femtosecond laser ablation. The guiding properties, micro-fluorescence and micro-Raman spectroscopy performance have been measured and discussed in detail.

\footnotetext{
* Corresponding author. Tel.: +86531 88363007; fax: +8653188363350.

E-mail address: drfchen@sdu.edu.cn (F. Chen).
} 


\section{Experiments in details}

The Yb:YAG crystal (doped by $15 \mathrm{at} \% \mathrm{Yb}^{3+}$ ions) used in this work was cut into dimensions of $10 \times 10 \times 2 \mathrm{~mm}^{3}$ and optically polished. Fig. 1 shows the schematic process for the Yb:YAG ridge waveguide fabrication. Firstly, one sample surface $\left(10 \times 10 \mathrm{~mm}^{2}\right)$ was irradiated with the fluence of $4 \times 10^{14}$ ions $/ \mathrm{cm}^{2}$ by using the 3 MV tandem accelerator at Helmholtz-Zentrum Dresden-Rossendorf, Germany. A planar waveguide layer was formed with the thickness of $\sim 8 \mu \mathrm{m}$ through the electronic damage of the irradiated region. The ion current density was kept at a lower level (around 6-8 $\mathrm{nA} / \mathrm{cm}^{2}$ ). After that, we used a Ti:Sapphire laser system (Spitfire, Spectra Physics), which generated linearly polarized pulses of $120 \mathrm{fs}$ with a central wavelength of $800 \mathrm{~nm}$ (with $1 \mathrm{kHz}$ repetition rate and $7 \mathrm{~mJ}$ maximum pulse energy) to microstructure the ridge waveguides on top of the previously irradiated planar waveguide surface. The sample was placed at a micropositioning $\mathrm{XYZ}$ motorized stage and the laser beam was focused by a $10 \times$ microscope (N.A. $=0.3$ ) at the surface of the crystal. The pulse energy (incident on sample) was set to $1.9 \mu \mathrm{J}$ by using a halfwave plate and a linear polarizer followed by a calibrated neutral density filter. Under these conditions, parallel ablation grooves were produced by scanning the sample at constant velocity. The peak fluence at focus with the selected pulse energy, $\sim 42 \mathrm{~J} / \mathrm{cm}^{2}$, was well above the typical values of ablation threshold for transparent dielectrics. We have used that value in order to get the walls of the ablation grooves as vertical as possible, but trying to minimize the damage of the surrounding material. Different scanning velocities and groove separations were used in order to investigate their effects on the propagation losses of the ridge waveguides. Table 1 shows the fabrication parameters of four waveguides (WG1-WG4) that we have investigated in detail in this work.

A polarized microscope (Axio Imager, Carl Zeiss) was used to inspect the fabricated ridge waveguides. A typical end-face coupling system was arranged to measure the modal profile of the guided modes by a He-Ne laser source at the wavelength of $632.8 \mathrm{~nm}$. The propagation losses of the Yb:YAG ridge waveguides were measured by using the back-reflection method [31].

In order to study the physical mechanism of $\mathrm{C}^{5+}$ ion irradiated Yb:YAG waveguide formation, a confocal micro-Raman spectrometer (Horiba/JobinYvon HR800) was used to measure the Raman properties and a Jasco U570 spectro-photometer was utilized to measure the micro-fluorescence spectra at room-temperature. A continuous-wave laser beam at wavelength of $473 \mathrm{~nm}$ was focused onto the end facet of the sample and the confocal micro-Raman spectra and micro-fluorescence spectra of the waveguide region and substrate region were measured, respectively.

\section{Results and discussion}

We calculated the electronic $\left(S_{\mathrm{e}}\right)$ and nuclear $\left(S_{\mathrm{n}}\right)$ stopping powers of the $15 \mathrm{MeV} \mathrm{C}^{5+}$ ion into Yb:YAG and the $\mathrm{C}^{5+}$ ion concentration distribution in the crystal by using the Stopping and Range of Ions in Matter (SRIM) 2010 code [32], as shown in Fig. 2(a). As it is indicated, in the range of $0-6 \mu \mathrm{m} S_{\mathrm{e}}$ peaks at $2.2 \mathrm{keV} / \mathrm{nm}$ at the depth of $6 \mu \mathrm{m}$, which is overwhelmingly dominant over $S_{\mathrm{n}}$, which remains zero in the first $0-6 \mu \mathrm{m}$ and the maximum value reaches $0.19 \mathrm{keV} / \mathrm{nm}$ at the depth of $8.5 \mathrm{~nm}$. It suggests that the lattice damage is mainly induced by electronic collisions rather than nuclear damage. Fig. 2(b) shows the reconstructed refractive index profile of the $\mathrm{C}^{5+}$ ion implanted planar waveguide. To determine the refractive index distributions of the waveguide, we utilized the $m$-line technique to measure the dark mode spectra. However, no dark modes were observed. This suggests that the waveguides are buried waveguide layers. Alternatively, we could roughly estimate the maximum refractive index change of the waveguide to be $\Delta n \approx 4 \times 10^{-3}$ by the numerical aperture method through the formula [9]

$\Delta n=\frac{\sin ^{2} \Theta_{\mathrm{m}}}{2 n}$

where $n=1.8297$ is the refractive index of the substrate, $\Theta_{\mathrm{m}}$ is the maximum incident angular deflection at which no transmitted power change occurs. By assuming the refractive index profile is similar to that of the $S_{\mathrm{e}}$ profile and the maximum index change is $\Delta n \approx 4 \times 10^{-3}$, we can reconstruct the $1 \mathrm{D}$ refractive index profile of the planar waveguide, as shown in Fig. 2(b).

Fig. 3(a) shows the image of the cross section of the ridge waveguide WG3 with the width of $30 \mu \mathrm{m}$ fabricated by fs-laser ablation of the $15 \mathrm{MeV} \mathrm{C}^{5+}$ ions irradiated Yb:YAG crystal. The 2D refractive index distribution (Fig. 3(b)) of the ridge waveguide is

Table 1

Propagation losses of the Yb:YAG ridge waveguides WG1-WG4 at $632.8 \mathrm{~nm}$.

\begin{tabular}{llll}
\hline Waveguide & Scan velocity $(\mu \mathrm{m} / \mathrm{s})$ & Width $(\mu \mathrm{m})$ & Propagation loss $\alpha(\mathrm{dB} / \mathrm{cm})$ \\
\hline WG1 & 15 & 30 & 3.9 \\
WG2 & 15 & 40 & 2.4 \\
WG3 & 15 & 50 & 2.1 \\
WG4 & 30 & 50 & 2.6 \\
\hline
\end{tabular}

b

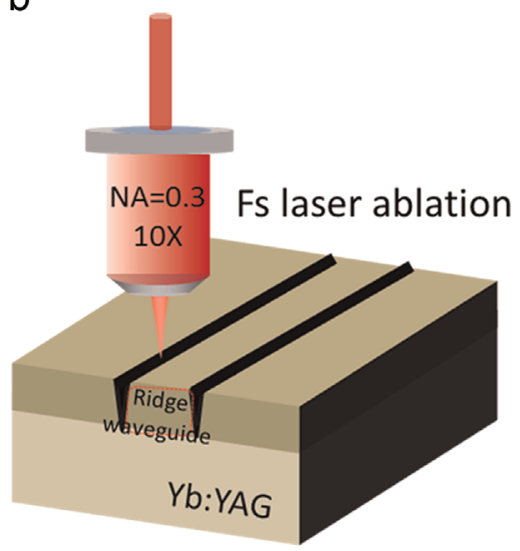

Fig. 1. Schematic of (a) $15 \mathrm{MeV} \mathrm{C}^{5+}$ ion irradiation and (b) femtosecond laser ablation process for the Yb:YAG ridge waveguide fabrication. 
therefore reconstructed considering the ridge waveguide geometry and the 1D planar waveguide index profile (Fig. 2(b)). Based on this refractive index profile, we can calculate the modal profile of the guided mode by the software Rsoft $^{\odot}$ Beam-Prop [33] based on the Finite Difference Beam Propagation Method (FD-BPM) [34] as shown in Fig. 3(d). Fig. 3(c) shows the measured near-field intensity distribution of fundamental mode for the ridge waveguide WG3. As one
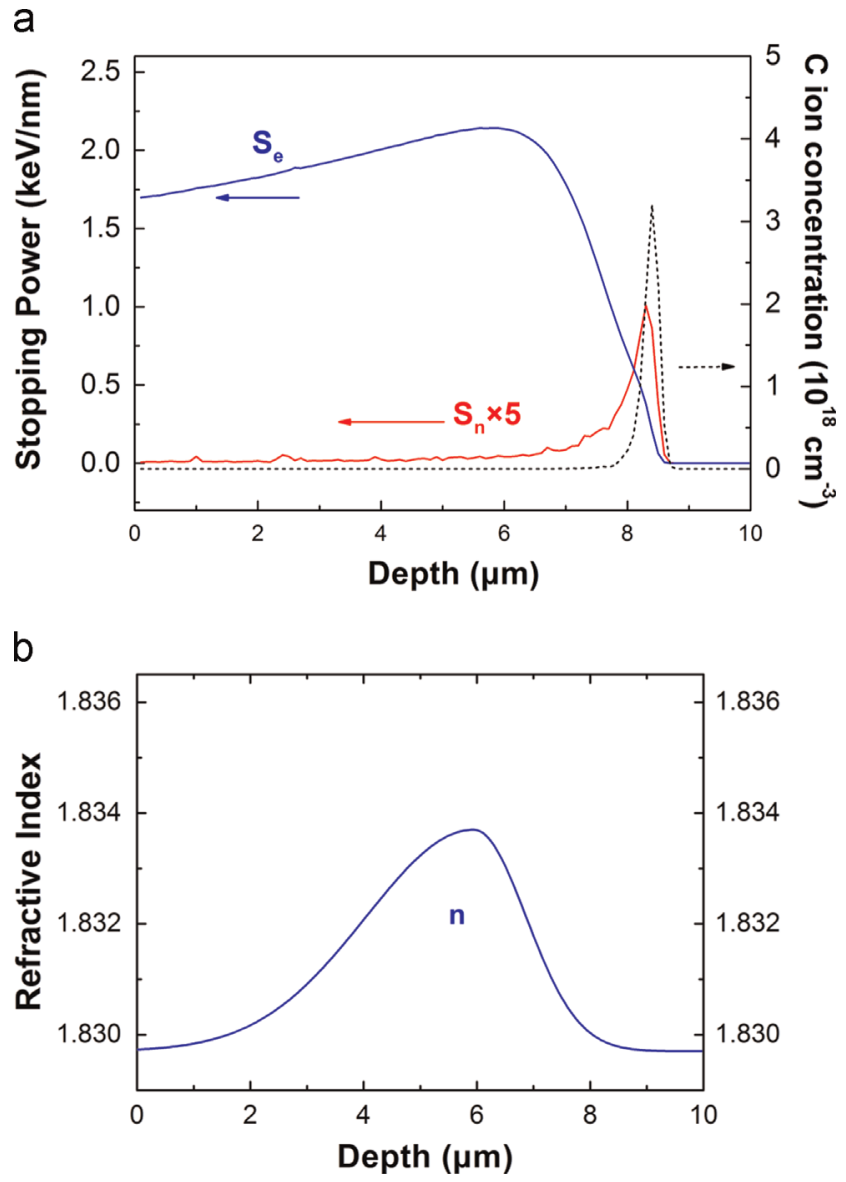

Fig. 2. (a) Electronic (blue line) and nuclear (red line) stopping powers and $C$ ion concentration as a function of the depth from the surface of the $15 \mathrm{MeV} \mathrm{C}^{5+}$ ion irradiated Yb:YAG crystal; and (b) the refractive index profile of the $\mathrm{C}^{5+}$ ion irradiated planar waveguide. (For interpretation of the references to color in this figure legend, the reader is referred to the web version of this article.) can see, the calculated distribution was in good agreement with the experimental result that confirms the reasonability of the refractive index map reconstruction.

Table 1 shows the propagation losses of the ridge waveguides WG1-WG4. WG1, WG2 and WG3 were fabricated with the same scan velocity $(15 \mu \mathrm{m} / \mathrm{s})$ but with different waveguide sizes (separation between the ridges $30 \mu \mathrm{m}, 40 \mu \mathrm{m}$ and $50 \mu \mathrm{m}$, respectively). As one can see, the propagation losses decrease as the ridge waveguide width increases. This behavior is mainly attributed to the roughness of the side-walls of ridge structure fabricated by fs-ablation, which results in the attenuation by scattering. Previous studies showed that for narrow ridge structures the sidewall scattering affection becomes more relevant [35]. WG3 and WG4 have the same waveguide width $(50 \mu \mathrm{m})$ but were fabricated with different scanning velocities $(15 \mu \mathrm{m} / \mathrm{s}$ and $30 \mu \mathrm{m} / \mathrm{s}$, respectively). Due to the large peak fluence during irradiation, the depth of the ablation grooves for both velocities was large enough to surpass the planar waveguide region. The measured propagation losses for both waveguides were $2.1 \mathrm{~dB} / \mathrm{cm}$ and $2.6 \mathrm{~dB} / \mathrm{cm}$, for WG3 and WG4 respectively. The main reason for the lower value of WG3 losses is that the roughness of the ablation side-wall decreases with the scanning velocity due to the "smoothing" effect of a larger number of incident pulses in each point of the sample. Thus, the losses due to scattering at the waveguide walls are reduced and the confinement is improved.

Fig. 4 depicts the confocal Raman spectra of the Yb:YAG crystal in the waveguide and substrate regions at room temperature. The sharp peaks are labeled and the frequency peaks from 300 to $900 \mathrm{~cm}^{-1}$ are $370,402,546,694,721,784$ and $859 \mathrm{~cm}^{-1}$. According to previous studies [36,37], the peaks at 370 and $402 \mathrm{~cm}^{-1}$ are attributed to the mixing mode of the lattice and $\nu_{3}$ mode of $\left(\mathrm{AlO}_{4}\right)$ unit. The peak at $546 \mathrm{~cm}^{-1}$ is assigned as the $\nu_{2}$ mode of $\left(\mathrm{AlO}_{4}\right)$ unit. The peaks at 694 , 721,784 and $859 \mathrm{~cm}^{-1}$ are attributed to the $\nu_{1}$ and $\nu_{4}$ mode of $\left(\mathrm{AlO}_{4}\right)$ unit. As we can see, the peak positions, peak intensities, and peak widths of the Raman spectra in the waveguide region had no obvious change with respect to that in the bulk region, which indicated that there was no large disorder or stress in the Yb:YAG crystal after swift $\mathrm{C}^{5+}$ ion irradiation.

Fig. 5 shows the obtained emission luminescence spectroscopy of the waveguide and bulk of Yb:YAG crystal. As we can see, twelve emission bands centered at 472.8, 476.6, 477.8, 478.9, 480.6, 481.5, 482.1, 485.5, 489.2, 491.1 and $493.1 \mathrm{~nm}$ could be observed in the wavelength range of $450-500 \mathrm{~nm}$. These bands are attributed to the cooperative up-conversion of $\mathrm{Yb}^{3+}$ pair of ions. In this phenomenon, the fluorescence is radiated through a transition of the excited a

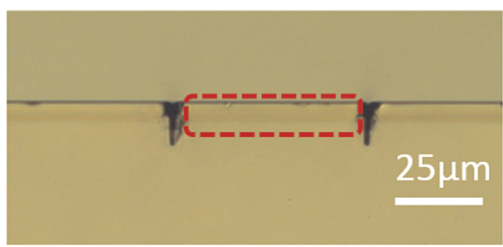

C

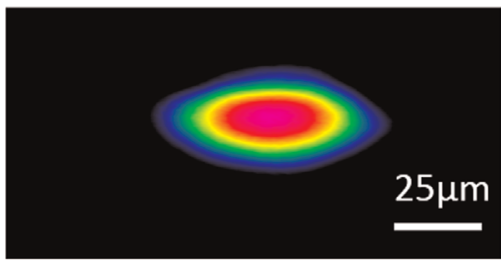

b

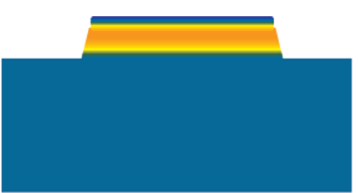

1.8337

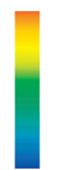

1.8297

d

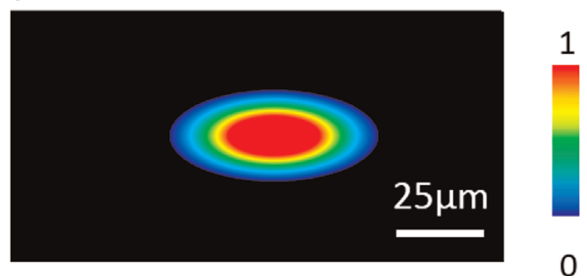

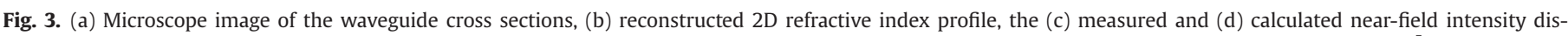

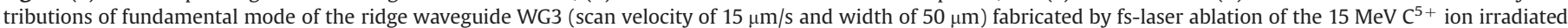
Yb:YAG crystal. 


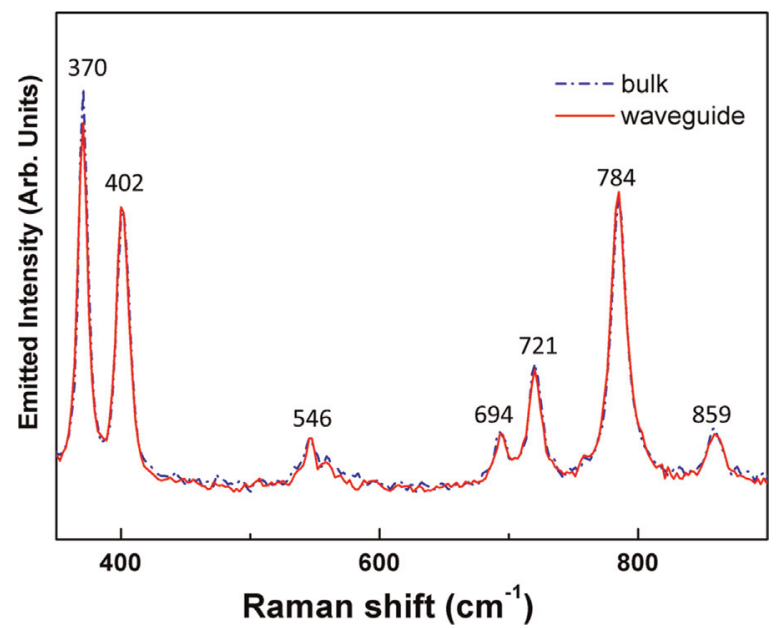

Fig. 4. Confocal micro-Raman spectra obtained from the waveguide region (red solid line) and substrate region (blue dashed line) in the Yb:YAG crystal.

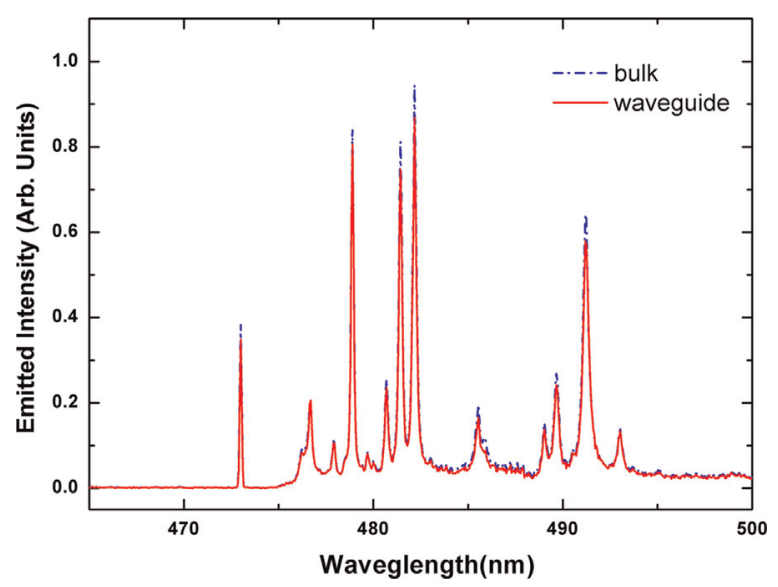

Fig. 5. The confocal micro-luminescence spectra of Yb3 + doped in YAG at room temperature in the waveguide region (red solid line) and substrate region (blue dashed line) in the Yb:YAG crystal.

coupled state ${ }^{2} \mathrm{~F}_{7 / 2}\left(\mathrm{Yb}^{3+}\right)$ to the ground coupled state ${ }^{2} \mathrm{~F}_{5 / 2}\left(\mathrm{Yb}^{3+}\right)$, which subsequently emitted a photon with the sum of energies and green light $[38,39]$. As one can see, the fluorescence intensity, line widths and spectral shifts of the line in the waveguide region has no obvious change compared to the bulk, which indicated that the original photoluminescence features of $\mathrm{Yb}^{3+}$ in $\mathrm{Yb}$ :YAG crystal have been preserved and the ion irradiation process has not caused microstructural changes in the Yb:YAG crystal.

\section{Conclusions}

We have reported on the fabrication of optical ridge waveguides in Yb:YAG crystal by using swift $\mathrm{C}^{5+}$ ion irradiation and subsequent femtosecond laser ablation. The minimum propagation loss of the ridge waveguides was measured to be $\sim 2.1 \mathrm{~dB} / \mathrm{cm}$. The confocal micro-fluorescence spectra showed that most of the $\mathrm{Yb}^{3+}$ luminescence features have been preserved and there was no significant stable modification of lattice, neither defects creation, after $\mathrm{C}^{5+}$ ion irradiation. In micro-Raman spectra, the peak positions and band widths had no obvious change, which demonstrated that no large lattice disorder or stress was generated after swift $C^{5+}$ ion irradiation.
In conclusion, the results demonstrate the optimum performance of Yb:YAG ridge waveguides fabricated with this combined technique and the potential applications as integrated laser sources.

\section{Acknowledgments}

The work is supported by the National Natural Science Foundation of China (No. U1332121) and Ministerio de Economía y Competitividad of Spain (FIS2013-44174-P). S.Z. acknowledges the funding by the Helmholtz-Gemeinschaft Deutscher Forschungszentren (HGF-VH-NG713). This work was partially carried out in CLPU facility (Lab. 2) in the framework of the access agreement concerning USAL staff.

\section{References}

[1] Murphy EJ. Integrated optical circuits and components: design and applications. New York: Marcel Dekker; 1999.

[2] Grivas C. Prog Quantum Electron 2011;35:159.

[3] Chen F, Vazquez de Aldana JR. Laser Photonics Rev 2014;8:251.

[4] Liu AC, Diagonnet MJF, Kino GS. Opt Lett 1994;19:466.

[5] Ostrowsky DB, Poirier R, Reiber LM, Deverdun C. Appl Phys Lett 1973;22:463.

[6] Belotti M, Galisteo-Lopez J, De Angelis S, Galli M, Maksymov I, Andreani LC, et al. Opt Express 2008;16:11624.

[7] Savatinova I, Savova I, Liarokapis E, Ziling CC, Atuchin VV, Armenise MN, et al. J Phys D 1998;31:1667.

[8] Zolotoyabko E, Avrahami Y, Sauer W, Metzger TH, Peisl J. Appl Phys Lett 1998; $73: 1352$.

[9] Siebenmorgen J, Petermann K, Huber G, Rademaker K, Nolte S, Tünnermann A. Appl Phys B 2009;97:251.

[10] Bain FM, Lagatsky AA, Thomson RR, Psaila ND, Kuleshov NV, Kar AK, et al. Opt Express 2009; 17:22417.

[11] Torchia GA, Meilán PF, Rodenas A, Jaque D, Mendez C, Roso L. Opt Express 2007; $15: 13266$

[12] Tan Y, Rodenas A, Chen F, Thomson RR, Kar AK, Jaque D, et al. Opt Express 2010;18:24994.

[13] Chen F. Laser Photonics Rev 2012;6:622.

[14] García-Navarro A, Olivares J, García G, Agulló-López F, García-Blanco S, Merchant C, et al. Nucl Instrum Methods Phys Res B 2006;249:177.

[15] Townsend PD, Chandler PJ, Zhang L. Optical effects of ion implantation. London: Cambridge University Press; 1994.

[16] Chen F, Wang XL, Wang KM. Opt Mater 2007;29:1523.

[17] Davis KM, Miura K, Sugimoto N, Hirao K. Opt Lett 1996;21:1729.

[18] Takaichi K, Yagi H, Lu J, Skirakawa A, Ueda K, Yanagitani T. Phys Status Solidi A 2003;200:R5

[19] Nakamura S, Yoshioka H, Matsubara Y, Ogawa T, Wada S. Opt Commun 2008;281:4411.

[20] Nakamura S, Yoshioka H, Matsubara Y, Ogawa T, Wada S. Jpn J Appl Phys 2009;48:060205.

[21] Pirri A, Alderighi D, Toci G, Vannini M. Opt Express 2009;17:23344.

[22] Dong J, Shirakawa A, Ueda K, Yagi H, Yanagitani T, Kaminskii AA. Opt Lett 2007;32:1890.

[23] Pirri A, Alderighi D, Toci G, Vannini M. Laser Phys 2010;20:931.

[24] Kaminskii AA. Laser crystals: their physics and properties. New York: SpringerVerlag; 1981.

[25] Brenier A, Boulon G. J Alloy Compd 2001;323:210.

[26] Kaminskii AA. Laser Photonics Rev 2007;1:93.

[27] Juodkazis S, Mizeikis V, Misawa H. J Appl Phys 2009;106:051101.

[28] Sugioka K, Cheng Y. Light Sci Appl 2014;3:e149.

[29] Jia Y, Rüter ChE, Akhmadaliev Sh, Zhou Sh, Chen F, Kip D. Opt Mater Express 2013;3:433.

[30] Jia Y, Vázquez de Aldana JR, Lu Q, Jaque D, Chen F. J Lightwave Technol 2013;31:3873.

[31] Ramponi R, Osellame R, Marangoni M. Rev Sci Instrum 2002:73:1117.

[32] Ziegler JF. Computer code SRIM. 〈http://www.srim.org $\rangle$.

[33] Rsoft Design Group. Computer Software BeamPROP version 8.0. 〈http://www. rsoftdesign.com>.

[34] Yevick D, Bardyszewski W. Opt Lett 1992;17:329.

[35] Yap KP, Delage AÉ, Lapointe J, Lamontagne B, Schmid JH, Waldron P, et al. J Lightwave Technol 2009;27:3999.

[36] Mace G, Schaack G, Toaning NG, Koningstein JA. Z Phys 1970;230:391.

[37] Schaack G, Koningstein JA. J Opt Soc Am 1970;60:1110.

[38] Nakazawa E, Shionoya S. Phys Rev Lett 1970;25:1710.

[39] Goldner Ph, Pellé F, Meichenin D, Auzel F. J Lumin 1997;71:137. 